\title{
Multiscale analysis of monoglyceride oleogels during storage
}

\author{
Kato Rondou ${ }^{1}$, Fien De Witte ${ }^{1}$, Tom Rimaux ${ }^{2}$, Wim Dewinter ${ }^{2}$, Koen Dewettinck ${ }^{1}$, Jan \\ Verwaeren $^{1}$, and Filip Van Bockstaele ${ }^{1}$ \\ ${ }^{1}$ Ghent University \\ ${ }^{2}$ Vandemoortele R\&D Centre
}

February 16, 2022

\begin{abstract}
Oleogelation offers the possibility to reduce the saturated fatty acid (SAFA) content while maintaining the desired organoleptic properties. Hereby, SAFA are replaced by other structurants which can create a three-dimensional network that immobilizes the liquid oil. Depending on the type of structurants, different structuring routes are identified. The use of monoglycerides (MAGs) as structurants is a promising approach thanks to their great self-assembling properties. However, implementation into the food industry is still hampered due to insufficient characterization. This research includes a multiscale analysis of two dynamically produced MAG-based oleogels as a function of the storage time (up to 8 weeks). Slight differences in the production process resulted in differences in techno-functional properties between the MAG-based oleogels MO1 and MO2. MO1 consisted of larger crystals, which resulted in a lower rigidity, lower stability and lower oil binding capacity compared to the other oleogel (MO2). On the nanoscale, it was found that the crystal nanoplatelets (CNPs) of MO1 contained a higher number of lamellae compared to the MO2. Additionally, the results obtained with ultra-small angle X-ray scattering indicated a larger equivalent diameter for the CNPs of MO1. As a function of the storage time, both oleogels did not show major structural changes up to 8 weeks of storage.
\end{abstract}

\section{Hosted file}

Main.text.file.MultiscaleAnalysisOfMonoglycerideOleogelsDuringStorage.docx available at https://authorea.com/users/460872/articles/556730-multiscale-analysis-of-monoglycerideoleogels-during-storage 

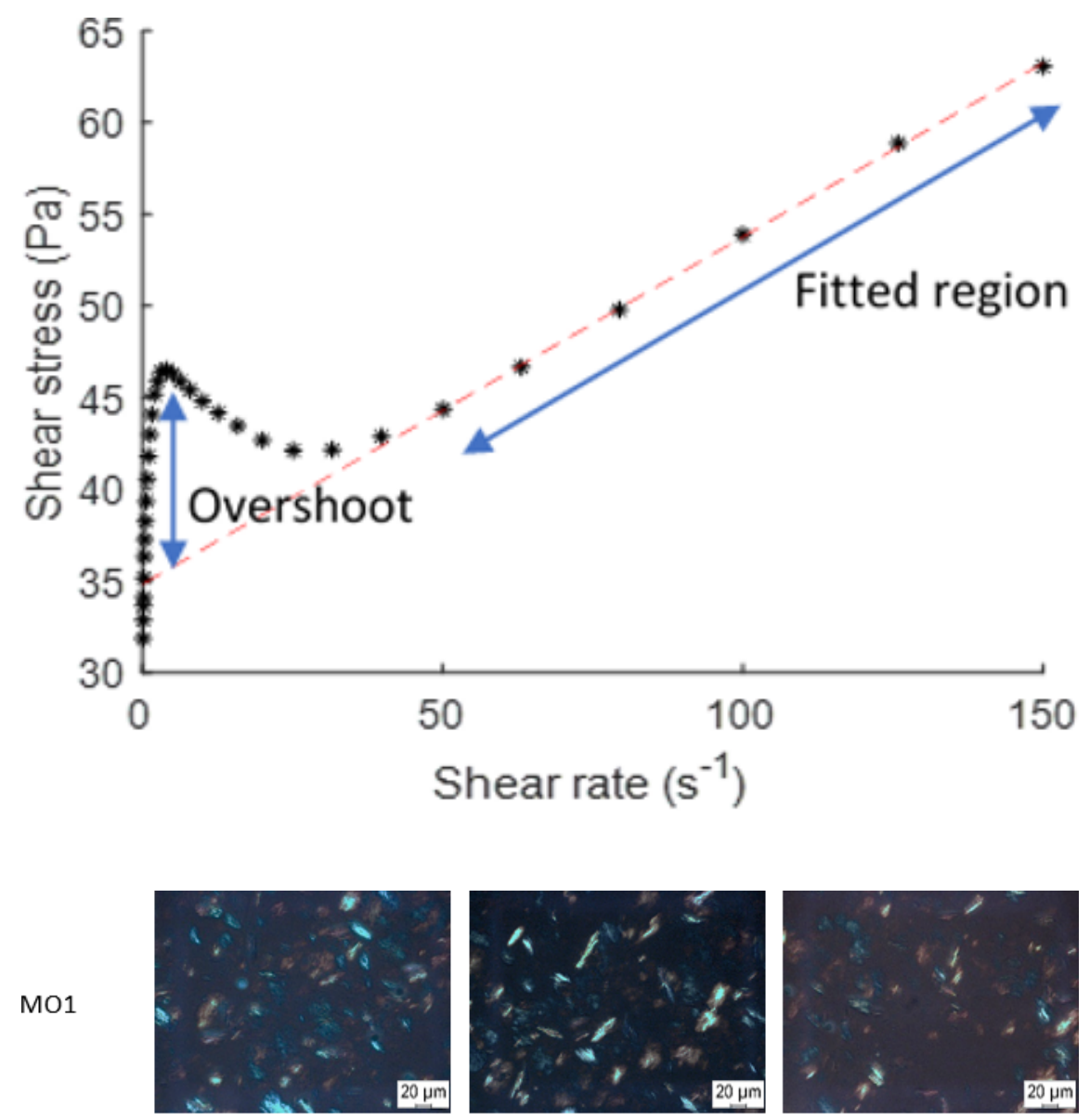

MO2

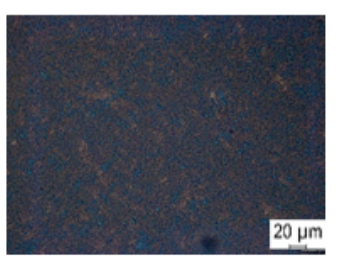

w1

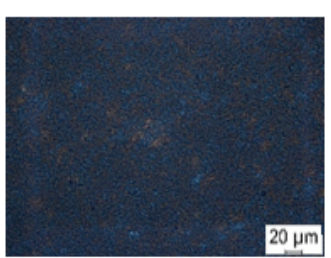

w4

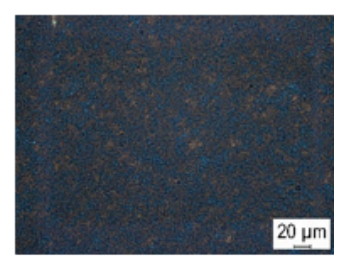

w8 

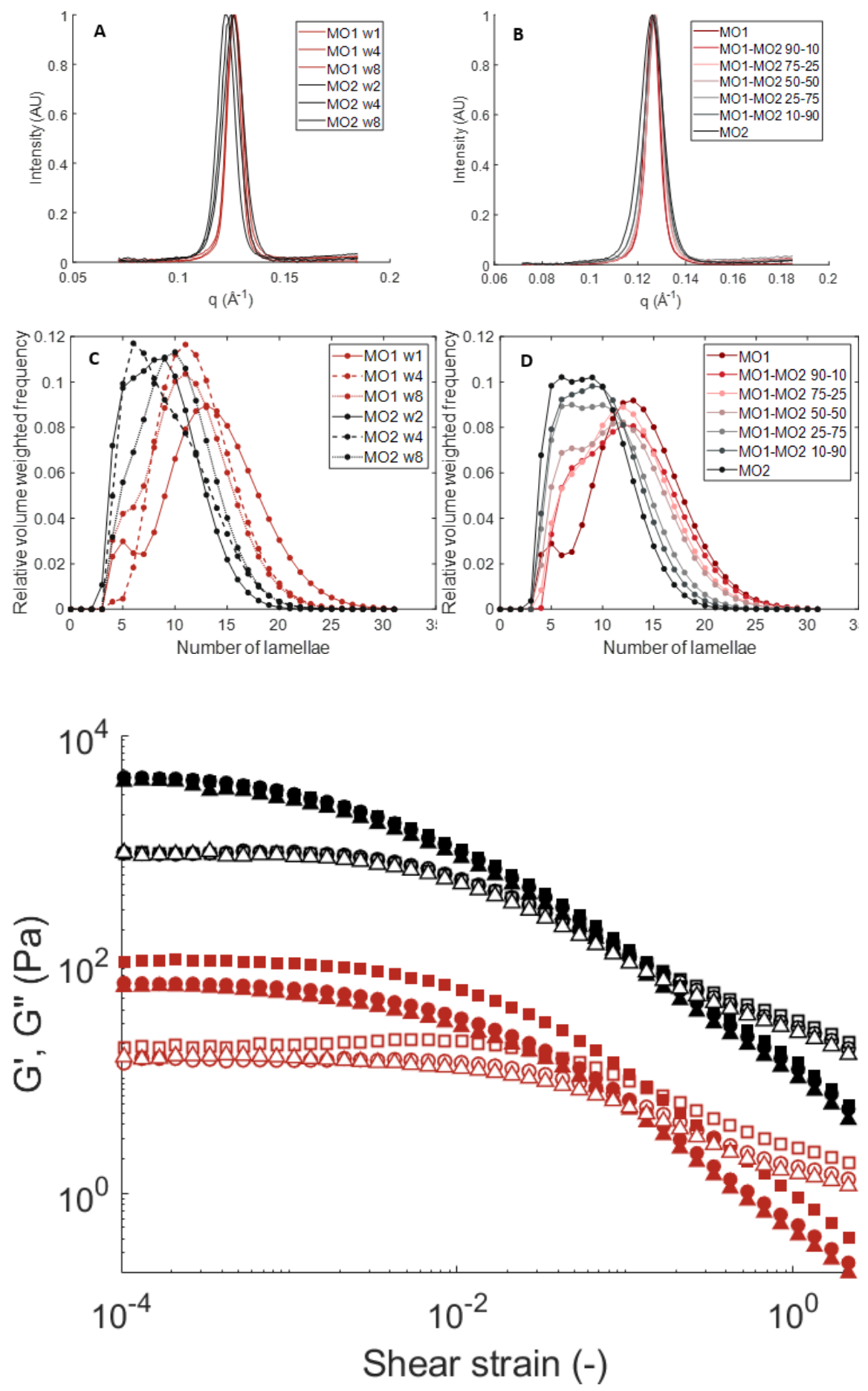

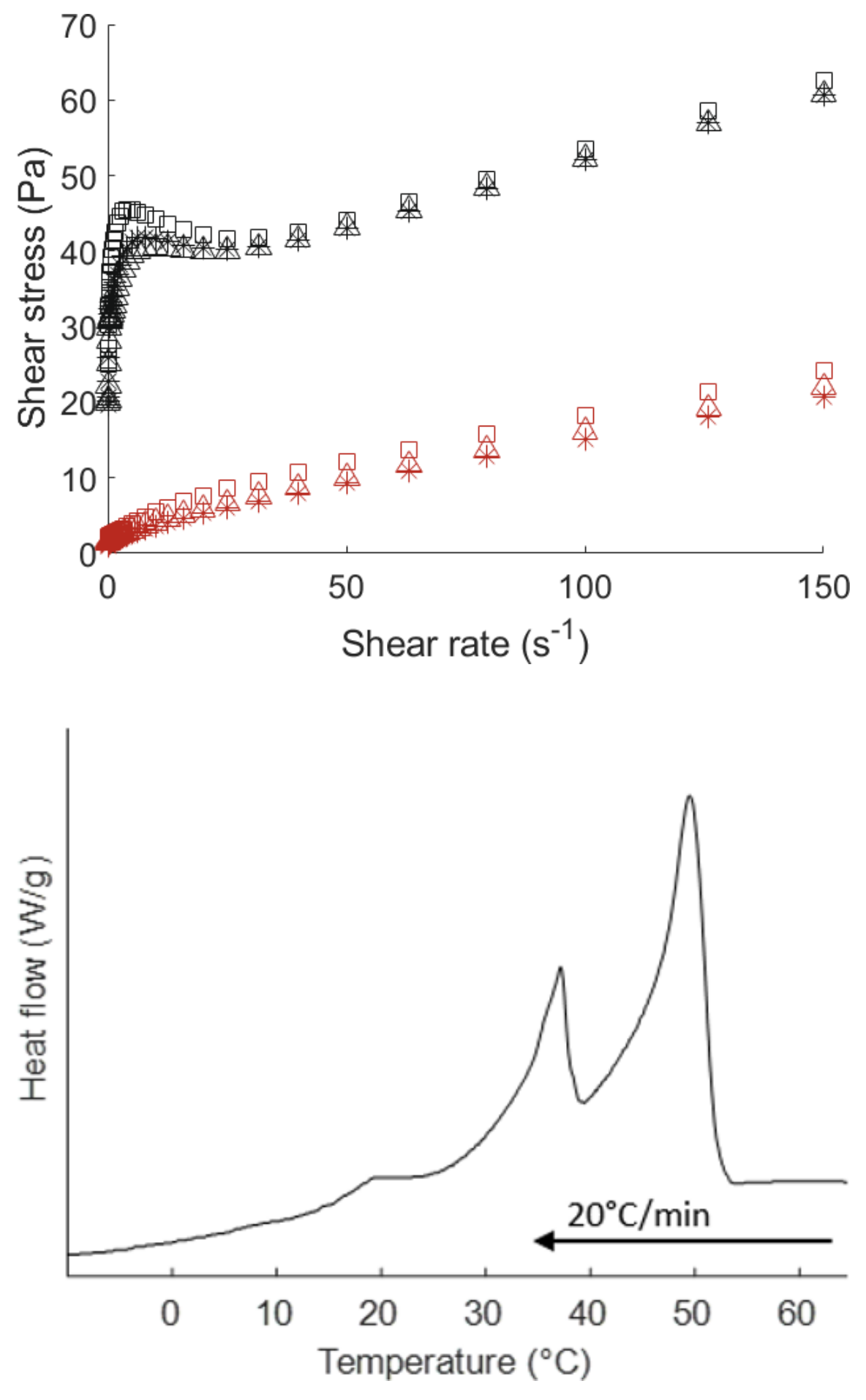


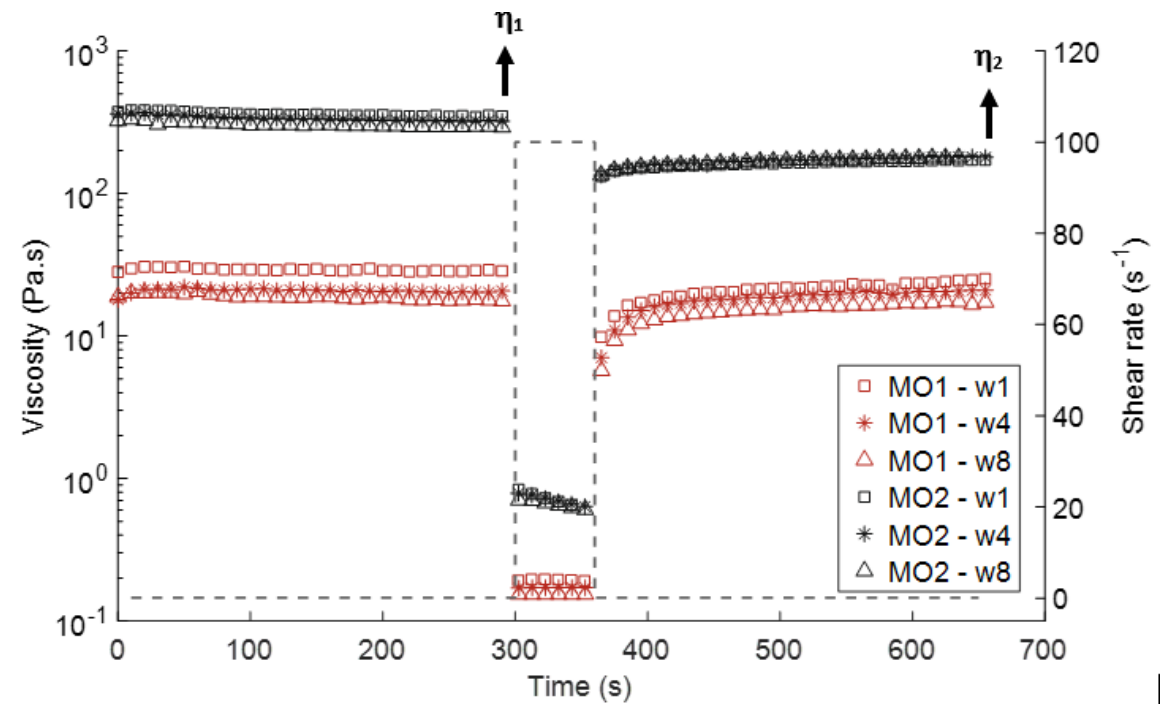

